Regiane Lima de Sousa

\author{
Desenvolvimento de Aplicações Sensíveis \\ ao Contexto usando Sistemas Multi-Agentes
}

Dissertação de Mestrado

Dissertação apresentada como requisito parcial para obtenção do título de Mestre pelo Programa de PósGraduação em Informática da PUC-Rio.

Orientadores: Carlos José Pereira de Lucena 
Regiane Lima de Sousa

\section{Desenvolvimento de Aplicações Sensíveis ao Contexto usando Sistemas Multi-Agentes}

Dissertação apresentada como requisito parcial para obtenção do título de Mestre pelo Programa de PósGraduação em Informática da PUC-Rio. Aprovada pela Comissão Examinadora abaixo assinada.

Prof. Carlos José Pereira de Lucena

Orientador

Departamento de Informática - PUC-Rio

Prof. Marco Antonio Casanova

Departamento de Informática - PUC-Rio

Prof. Ricardo Choren

Departamento de Informática - IME

Prof. Firmo Freire

Departamento de Informática - PUC-Rio

Prof. José Eugenio Leal Coordenador Setorial do Centro

Técnico Científico - PUC-Rio 
Todos os direitos reservados. É proibida a reprodução total ou parcial do trabalho sem autorização da universidade, da autora e dos orientadores.

\section{Regiane Lima de Sousa}

Graduou-se no Curso de Bacharelado em Ciência da Computação da Universidade Federal do Pará (UFPA) em 2003. Hoje atua como analista de sistemas prestando serviços para o TRT $1^{\text {a }}$ Região.

Ficha Catalográfica

Sousa, Regiane Lima

Desenvolvimento de aplicações sensíveis ao contexto usando sistemas multi-agentes / Regiane Lima de Sousa ; orientador: Carlos José Pereira de Lucena. 2007.

64 f. : il. ; $30 \mathrm{~cm}$

Dissertação (Mestrado em Informática)-Pontifícia Universidade Católica do Rio de Janeiro, Rio de Janeiro, 2007.

Inclui bibliografia.

1. Informática - Teses. 2. Aplicações sensíveis ao contexto. 3. Engenharia de software para sistemas multiagentes. 4. Reengenharia. 5. Frameworks. 6. Avaliação. I. Lucena, Carlos José Pereira de. II. Pontifícia Universidade Católica do Rio de Janeiro. Departamento de Informática. III. Título. 


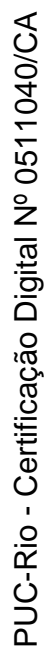

À minha família 


\section{Agradecimentos}

A Deus, por ter me guiado continuamente nesta jornada. À minha família, principalmente ao meu marido Roberto, por todo apoio, motivação e compreensão.

Ao professor Lucena, por ter me honrado com sua experiência profissional, particularmente, nos trâmites acadêmicos. Ao professor Casanova, por todas as palavras de incentivo, apoio e confiança. Ao Uirá pelas palavras motivadoras, ensinamentos e direcionamentos ao longo do mestrado.

Aos meus amigos Kleder, Melina, Léo, Cidiane, Karla e Klessis, por terem aceitado o desafio de ingressar na carreira acadêmica no Rio de Janeiro. À Cidiane, particularmente, por ter estado ao meu lado em momentos difíceis desde minha chegada ao Rio. Aos amigos do LAC, em especial ao José Viterbo, que compartilhou comigo o interesse no tema escolhido para esta Dissertação.

Aos meus colegas de Mestrado José Guedes, Caculé, Marcelo Malcher, Vinci, Aldo, Glória, Maíra Gatti, Ana Carolina e Felipi, que tornaram o dia a dia na PUC mais agradável. 


\section{Resumo}

Sousa, Regiane. Desenvolvimento de Aplicações Sensíveis ao Contexto usando Sistemas Multi-Agentes. Rio de Janeiro, 2007. 64p. Dissertação de Mestrado - Departamento de Informática, Pontifícia Universidade Católica do Rio de Janeiro.

O desenvolvimento de aplicações sensíveis ao contexto (ASCs) constitui-se como uma tarefa não-trivial em conseqüência das características relacionadas à sensibilidade ao contexto, como abertura, comunicação assíncrona e falta de mecanismos modulares na propagação de informações de contexto. Por outro lado, um paradigma de desenvolvimento de software é considerado uma ferramenta básica para a construção de sistemas de software. Particularmente, a Engenharia de Software para Sistemas Multi-Agentes (ESSMA) tem se apresentado como paradigma promissor para o desenvolvimento de aplicações distribuídas, abertas e extensíveis. Agentes de software são elementos cuja execução leva ao alcance dos objetivos de um sistema através de suas propriedades de interação, adaptação e autonomia. Partindo destas características, é possível propor soluções para o desenvolvimento de ASCs que facilitem a satisfação dos requisitos mencionados acima. Este trabalho tem dois objetivos principais: (1) apresentar estudos de caso envolvendo o uso de ESSMA no desenvolvimento de ASCs e (2) propor um framework como ferramenta de reuso de sensibilidade ao contexto nas aplicações. A avaliação dos estudos e da proposta do framework é utilizada para a demonstração da usabilidade de ESSMA em ASCs. A avaliação dos estudos é efetuada através da generalização de resultados, além da aplicação de métricas com foco em atributos, como a modularidade.

\section{Palavras-chave}

Aplicações Sensíveis ao Contexto, Engenharia de Software para Sistemas Multi-Agentes, Reengenharia, Frameworks, Avaliação. 


\section{Abstract}

Sousa, Regiane. Context-Aware Application Development using MultiAgent Systems. Rio de Janeiro, 2007. 64p. Master Thesis - Computer Science Department, Pontifical Catholical University of Rio de Janeiro.

The development of context-aware applications (CAAs) is not a trivial task due to their intrinsic features, such as openness, asynchrony communication, and the lack of modular abstractions and mechanisms for the propagation of context information. On the other hand, a software development paradigm is actually considered a basic tool for the construction of any software system. In particular, the Software Engineering for Multi-Agent Systems (SEMAS) is often introduced as a promising paradigm for the development of distributed, open, and extensible applications. Software agents are elements whose execution leads to the reach of the system's goals, through their interaction, adaptation, and autonomy properties. From the agent properties, it becomes possible to provide solutions for the development of CAAs in order to facilitate the satisfaction of the common requirements mentioned above. This work has two main purposes: (1) the development of case studies involving the use of SEMAS for three CAA-specific applications; (2) propose a framework to support the reuse of context-awareness features in the CAAs. The evaluation of the case studies and of the framework is used for the demonstration of SEMAS usability in the CAA-specific domain. Some evidences about the generality of the results are also provided, beyond the quantitative measurements based on common quality attributes, such as the modularity.

\section{Keywords}

Context-aware Applications, Software Engineering for Multi-Agent Systems, Reengineering, Frameworks, Evaluation. 


\section{Sumário}

1 Introdução 13

1.1. Problema 13

1.2. Limitações das Soluções Existentes 15

$\begin{array}{ll}\text { 1.3. Solução Proposta } & 18\end{array}$

1.4. Objetivos 19

1.5. Organização do Texto 20

2 Aplicações Sensíveis ao Contexto $\quad 21$

2.1. Definições Básicas de ASCs 21

2.1.1. Contexto e ASCs 22

2.1.2. Serviços e APIs de MoCA 23

2.2. ASCs Baseadas em MoCA 25

2.2.1. Virtual Lines $\quad 25$

2.2.2. Wireless Marketing Service 30

2.3. Análise do Uso Direto das APIs de Middlewares em ASCs 33

3 Engenharia de Software para Sistemas Multi-Agentes 35

3.1. Definições Básicas de ESSMA 35

3.1.1. Elementos do Conhecimento de Agentes 36

3.1.2. Tipos de Agentes 36

$\begin{array}{ll}\text { 3.1.3. Propriedades de Agência } & 37\end{array}$

3.1.4. Serviços e API de JADE 38

3.2. Reengenharia de ASCs usando JADE 40

3.2.1. Virtual Lines 40

3.2.2. Wireless Marketing Service 43

3.3. Análise do Uso de ESSMA na Reengenharia de ASCs 44

4 Reuso em ASCs usando ESSMA: o Framework CAAF 46

4.1. Estrutura de CAAF 46

4.2. Exemplo de Instanciação de CAAF 49 
5 Avaliação Quantitativa dos Estudos de Caso 53

5.1. Procedimentos e Métricas 53

5.2. Resultados Encontrados 54

6 Conclusão 57

6.1. Trabalhos Relacionados 57

6.2. Contribuições do Trabalho 59

6.3. Trabalhos Futuros 60

$\begin{array}{ll}\text { Referências Bibliográficas } & 61\end{array}$ 


\section{Lista de Figuras}

Figura 1. Dependências entre as APIs de MoCA 15

Figura 2. Arquitetura de CoBrA 16

Figura 3. Arquitetura ACAI 16

Figura 4. Arquitetura MoCA (Sacramento et al, 2004) 24

Figura 5. Classes de Negócio da Aplicação Virtual Lines 26

Figura 6. Módulo Cliente da Aplicação Virtual Lines 27

Figura 7. Módulo Servidor da Aplicação Virtual Lines 28

Figura 8. Cenário de Criação de Usuário em VL 29

Figura 9. Cenário de Solicitação de uma Reserva em VL 29

Figura 10. Manipulação de Contexto de Localização em Virtual Lines 30

Figura 11. Arquitetura da Aplicação Wireless Marketing Service (LAC) 31

Figura 12. Módulo Servidor da Aplicação Wireless Marketing Service 32

Figura 13. Módulo Cliente da Aplicação Wireless Marketing Service 32

Figura 14. Cenário de Notificação de Localização de Usuário em WMS 33

Figura 15. Reengenharia do Módulo Cliente de VL usando ESSMA 41

Figura 16. Reengenharia do Módulo Servidor de VL usando ESSMA 42

Figura 17. Cenário de Criação do Usuário em VL 42

Figura 18. Cenário de Solicitação de uma Reserva em VL 43

Figura 19. Reengenharia do Módulo Servidor de WMS usando ESSMA 43

Figura 20. Reengenharia do Módulo Cliente de WMS usando ESSMA 44

Figura 21. Classes do Framework CAAF para o Servidor da Instância 47

Figura 22. Classes do Framework CAAF para o Cliente da Instância 48

Figura 23. Diagrama de Classes da Aplicação Servidora de HealthCare49

Figura 24. Diagrama de Classes da Aplicação Cliente de HealthCare 50

Figura 25. Cenário de Envio de Pacientes com Prioridade em HC 51 


\section{Lista de Tabelas}

Tabela 1. Métricas para Avaliação dos Estudos e do Framework 54

Tabela 2. Valores de Acoplamento, Coesão e Tamanho em VL 55

Tabela 3. Valores de Acoplamento, Coesão e Tamanho em WMS 55 


\section{Lista de Siglas e Abreviaturas}

ACAI

API

ASC

CoBrA

CAAF

ESSMA

$\mathrm{HC}$

MoCA

$\mathrm{OO}$

SMAs

VL

WMS
Agent-Based Context-Aware Infrastructure

Application Programming Interface

Aplicação Sensível ao Contexto

Context Broker Architecture

Context-Aware Application Framework

Engenharia de Software para Sistemas Multi-Agentes

Health Care

Mobile Collaboration Architecture

Orientação a Objetos

Sistemas Multi-Agentes

Virtual Lines

Wireless Marketing Service 